\title{
La correspondance Rougemont-Paulhan
}

\section{Krisztina Horváth}

\section{(2) OpenEdition}

Journals

Édition électronique

URL : https://journals.openedition.org/edl/1854

DOI : 10.4000/edl.1854

ISSN : 2296-5084

\section{Éditeur}

Université de Lausanne

\section{Édition imprimée}

Date de publication : 15 décembre 2019

Pagination : 85-100

ISBN : 978-2-940331-72-7

ISSN : 0014-2026

\section{Référence électronique}

Krisztina Horváth, «La correspondance Rougemont-Paulhan », Études de lettres [En ligne], 311 | 2019, mis en ligne le 15 décembre 2021, consulté le 17 décembre 2021. URL : http://

journals.openedition.org/edl/1854; DOI : https://doi.org/10.4000/edl.1854

Ce document a été généré automatiquement le 17 décembre 2021.

(c) Études de lettres 


\title{
La correspondance Rougemont- Paulhan
}

\author{
Krisztina Horváth
}

Il n'est pas sans agrément de découvrir qu'une
opinion commune est fausse.
J. Paulhan, Les fleurs de Tarbes

1 La correspondance de Denis de Rougemont et de Jean Paulhan, comme l'histoire de la Nouvelle Revue française (NRF) de Paulhan, se scinde en deux périodes distinctes qui correspondent, dans la trajectoire de l'un comme de l'autre, à des phases différentes de leur biographie intellectuelle: les lettres témoignent de circonstances, de préoccupations, de goûts, de styles et même de compagnonnages fort différents et peuvent être considérées comme de précieux documents pour l'histoire de la vie intellectuelle européenne $\mathrm{du} \mathrm{xx}^{\mathrm{e}}$ siècle. Si plusieurs colloques ont été consacrés à l'histoire de la NRF, et en particulier à celle de Jean Paulhan ${ }^{2}$, la figure de Denis de Rougemont n'y est que rarement évoquée; et pourtant, la relation entre les deux écrivains est intéressante à de nombreux égards.

2 Nous allons présenter cette correspondance en deux liasses fictives de lettres, suivant l'ordre chronologique : la première va de la prise de contact en 1926, et des premières contributions de Denis de Rougemont en 1931, jusqu'à la guerre et au retrait de Paulhan de la NRF en juin 1940 ; la seconde comprend un corpus qui s'échelonne de 1946 à 1961. Du formel « Monsieur » de la première lettre de Paulhan jusqu'au tendre « Cher Denis » de la dernière, chaque période a ses particularités et l'écriture épistolaire évolue : le ton devient plus direct, Rougemont et Paulhan parlent désormais en compagnons de longue date, les débats deviennent plus âpres, les confessions plus intimes, le style moins formel. 


\section{L'entre-deux-guerres}

\subsection{La prise de contact}

3 La prise de contact, formelle et cordiale, se fait par Jean Paulhan dès $1926^{3}$, suite à la parution d'un texte de Denis de Rougemont dans la Bibliothèque universelle et Revue de Genève $^{4}$ : il ne lui offre rien de moins que de collaborer à la NRF. Paulhan, depuis la mort de Jacques Rivière en février 1925, dirige la revue «sous la tutelle trompeuse ${ }^{5}$ » de Gide : rédacteur en chef jusqu'en 1935, puis directeur, il est sans cesse à la recherche de nouvelles plumes, mais il impose rarement des notes 6 . Il propose des comptes rendus, mais attend surtout des propositions de la part de ses collaborateurs.

Denis de Rougemont a probablement décliné cette première offre de manière courtoise : la modestie de sa réponse manuscrite ${ }^{7}$ laisse deviner, malgré son jeune âge, la conscience d'une mission plus importante à venir. La reconnaissance dont témoigne la lettre de Paulhan a cependant une grande valeur à ces yeux : Rougemont est déjà lié avec les milieux littéraires et intellectuels, et la NRF sera pour lui une tribune de choix. Ainsi, de 1931 à 1940, comme l'observe Alain Corbellari ${ }^{8}$, Rougemont a écrit dans tous les volumes de la NRF, excepté quelques mois entre mai 1934 et août 1935, correspondant à la fin de son séjour en tant que « chômeur intellectuel » sur l'île de Ré puis dans le Gard.

Cinq années s'écouleront entre ce bref échange de 1926 et la première publication de Denis de Rougemont dans les pages de la NRF. Années de formation et de voyages qui verront naître des textes importants, Les méfaits de l'instruction publique (1929), Le paysan $d u$ Danube (1932), et pendant lesquelles Rougemont publie dans d'autres revues: ses articles et comptes rendus paraissent ainsi régulièrement dans la Bibliothèque universelle et Revue de Genève et dans la Revue de Belles-Lettres.

\subsection{Le « tournant de 1932 » et le Cahier de revendications}

6 La correspondance est aussi un précieux document-témoignage du «tournant de 1932 » et d'une « conscience de génération commune à des jeunes gens qui ont vécu la guerre, mais qui ne l'ont pas faite ${ }^{9}$ ". En témoigne l'élaboration du Cahier de revendications, numéro spécial de la NRF paru le $1^{\text {er }}$ décembre 1932, et coordonné en deux mois seulement par Denis de Rougemont.

7 Paulhan, adepte du paradoxe et des contradictions, avait pourtant émis quelques doutes quant à l'ambition de "Cause commune", sorte d'article-manifeste de Rougemont paru l'été précédent dans la revue Présence. "Croyez-vous qu'il soit prudent, ingénieux ou sage de se mettre à dix-huit pour découvrir la vérité? $»^{10}$, demandait-il, juste avant de proposer à Rougemont de se charger de la rédaction de ce numéro offrant une tribune à des intellectuels issus de différents horizons de contestations, personnalistes et marxistes ${ }^{11}$.

8 C'est Paulhan qui décide du titre de Cahier de revendications. S'il propose à la première lecture de retirer les textes de Jean Sylveire et d'Alexandre Marc, Rougemont se refuse à les supprimer ${ }^{12}$. La neutralité éditoriale de Paulhan s'illustrera par quelques banales suggestions orthographiques ("rien de moins » que au lieu de « rien moins que », nous en avons «davantage» au lieu de «nous en avons plus»), dont Rougemont tiendra 
compte dans sa postface, "À prendre ou à tuer ", mais aucune remarque de fond au sujet de ce texte virulent, ce qui vaut presque une approbation.

9 Après la parution, Paulhan transmet à Rougemont, avec un brin de provocation, les premiers échos que rencontre le numéro :

Il se dégage des nouvelles lettres que je reçois à peu près la réaction suivante : « $\mathrm{Ce}$ que dit Denis de Rougemont est très bien mais cela pourrait sembler dans un an tout à fait ridicule. Qu'est-il disposé à faire dès maintenant? $»^{13}$

De son côté, Rougemont observe :

Je recueille les réactions les plus diverses, pour et contre. Mais rien du côté communiste, sauf une réfutation de détail, douce et polie, de mon article, par Nizan au cours d'une conférence récente.

La question «que compte-t-il faire dès maintenant?» traduit, je crois, plus de "curiosité» bourgeoise et d'impatience indiscrète que de véritable volonté d'action. On ne peut rien faire d'autre, hic et nunc, que ce que nous faisons : préparer un terrain, une doctrine, une ambiance; pousser quelques analyses un peu brutales ${ }^{14}$.

Nizan critiquait notamment le déséquilibre du Cahier, au dépens des marxistes. À la suite de cette réfutation "douce et polie", le ton va bientôt monter: Rougemont annonce ainsi à Paulhan avoir répondu dans la revue Europe par une lettre ouverte ${ }^{15}$ (qui sera suivie d'une riposte de Nizan ${ }^{16}$ ).

J'ai répondu [que Nizan] a connu la composition et l'esprit du Cahier avant d'y contribuer, et qu'il vient un peu tard pour faire le petit pur ${ }^{17}$.

11 Ajoutons que Nizan fut certainement moins choqué par la simple composition du Cahier que mis dans l'embarras par la véhémence de la postface de Rougemont ${ }^{18}$.

En tout cas, les réactions transmises par Paulhan ne font que conforter Rougemont dans l'idée d'un second Cahier qui donnerait «des précisions d'action, au lieu de se borner à décrire des fondements idéologiques "; Paulhan lui-même semble retenir cette idée même si finalement, elle n'aboutit pas.

\subsection{Rester présent dans le paysage intellectuel parisien}

13 Après l'annonce du mariage de Rougemont avec Simonne Vion au début du mois de juillet 1933, Paulhan les invite chaleureusement à séjourner à Port-Cros ${ }^{19}$. En octobre, Rougemont remercie le couple Paulhan pour ce séjour à La Vigie et annonce sa décision de quitter pour un temps la capitale :

La beauté de votre Vigie nous a d'ailleurs donné l'idée, l'envie et enfin la volonté de vous imiter, et nous allons partir d'ici vers le 20 octobre, pour passer l'hiver dans l'̂̂le de Ré, où ma belle-mère possède une petite maison. Je Sers ayant définitivement suspendu ses services, et les miens, c'est à peu près la seule solution qui nous reste. Je n'en suis pas du tout fâché. Vous ne tarderez pas à lire les conséquences de tout cela ${ }^{20}$.

14 La maison d'édition "Je sers ", qui employait comme secrétaire le jeune écrivain, ne disparut pas complètement, mais son dépôt de bilan mit fin à la "relative sécurité matérielle» du couple. Rougemont se retrouva alors dans une situation précaire, l'argent qu'il gagnait de sa plume ne lui permettant plus de continuer de vivre à Paris ${ }^{21}$.

Les lettres de novembre 1933 témoignent toutefois d'une volonté de rester "présent " dans le paysage intellectuel parisien. Paulhan continue d'ailleurs d'associer Rougemont à la vie de la revue, lui faisant part du projet d'y inclure une rubrique consacrée aux films du mois, aux pièce de théâtre, aux expositions et aux livres d'art, aux concerts, 
bref «aux sorties» parisiennes, et même aux faits-divers, aux conversations, aux événements de la vie intime ou encore aux événements politiques, "ce qui serait diablement difficile ». Et de solliciter Rougemont pour envoyer de temps en temps de telles notes à caractère politique. Comme souvent, Paulhan ajoute une information essentielle en post scriptum, à la main: «Un an plus tard me semblerait un titre parfait ${ }^{22}$ ", faisant ici allusion à une suite au Cahier de revendications. Mais aucun texte de Rougemont ne parut dans la NRF sous ce titre, et l'idée d'une suite au Cahier fut définitivement abandonnée après la crise du 6 février 1934, visiblement aussi parce que les préoccupations de Rougemont étaient déjà ailleurs :

Ce qui serait intéressant, ce serait de faire une autre chronique, dans quelques mois, sur le seul Ordre Nouveau, qui se développe assez puissamment. De toutes façons, je reste redevable à la Revue d'une chronique déjà payée ${ }^{23}$ !

Rougemont exprime par ailleurs ses doutes quant à l'idée de la rubrique «carnet des spectateurs » et surtout celle de la chronique "faits-divers ", et lui oppose le pluralisme de la NRF :

Quant aux faits-divers, conversations et intimité, leur critique ne suppose-t-elle pas une Weltanschauung bien définie, un point de vue éthique et politique tout au moins? Je ne dis pas que la NRF n'en ait pas ; mais elle en a plusieurs ${ }^{24}$.

Le contact est donc vivant et Rougemont semble impliqué dans la vie même de la revue. Mais l'espacement des lettres et quelques projets de publication avortés ${ }^{25}$ laissent trahir, malgré l'aubaine inattendue d'un prix Schiller pour Le paysan du Danube, l'humeur de moins en moins optimiste de cette expérience «du vivre de peu » dont nous pouvons suivre de près l'évolution dans Le journal d'un intellectuel en chômage.

De Francfort, où il enseigne à l'Université de novembre 1935 à juin 1936, Rougemont informe Paulhan des faibles effectifs inscrits en français ( $« \mathrm{Il}$ y a fort peu d'étudiants cette année - environ le dixième de ce qu'il y en avait en $1932 »^{26}$ ), des livres confisqués à la frontière et des librairies manquant déjà de livres français.

En 1938, un roman soumis à Gallimard, La Folle Vertu, que Rougemont décide finalement de retirer malgré l'avis favorable de Paulhan, crée un peu de froid entre les deux hommes ${ }^{27}$. Paulhan s'était dit «préoccupé » par ce texte qu'il trouvait attachant dans son imperfection :

Peut-être aurais-je voulu qu'il y eût une « suite » plus précise à certaines réflexions de la première partie. (Mais il est certain que le roman n'existe qu'au prix d'une distraction). [...] Mais c'est une très belle œuvre, à laquelle je n'ai pas fini de songer ${ }^{28}$.

La remarque est caractéristique de Paulhan : plusieurs témoignages évoquent son goût pour le paradoxe, sa manière de réunir dans la même phrase des affirmations contraires, louer l'imperfection, dénoncer la perfection. " Paulhan connaissait si bien l'orgueil et la susceptibilité des écrivains qu'il ne se privait pas d'en jouer » observe Laurence Brisset ${ }^{29}$. Rougemont, apparemment, ne jouait pas à ce jeu-là, trop pressé de publier un texte autrement plus « utile $»^{30}$, faisant ici allusion à son Journal d'Allemagne. Il retira donc $L a$ Folle Vertu de la publication, ce dont il s'excusa dans une lettre datée du 29 juillet 1938.

21 En 1938-1939, la correspondance devient majoritairement d'ordre «technique ». Rougemont est souvent en retard avec ses « Airs du mois » qui visiblement le lassent un peu, propose d'autres contributeurs pour des billets que l'on attendait de lui, s'embarrasse pour un texte jugé imparfait pour un numéro d'hommage à Gide, 
demande le renvoi de l'épreuve. Il fait part de ses doutes dans plusieurs missives, avant d'envoyer sa version définitive qui est accompagnée d'une lettre datée du 24 août 1939 :

J'ai ajouté à ma chronique quatre pages importantes sur la contradiction entre l'esthétique et l'éthique de Gide - l'une puritaine, l'autre libérée - contradiction où réside sa tension personnelle, sa personne au sens où je l'entends ${ }^{31}$.

\subsection{La parenthèse de la guerre} dans des circonstances dramatiques : la dernière lettre de Paulhan datée du 22 janvier 1940 donne des nouvelles télégraphiques de certains écrivains, de Malraux qui s'engage, d'Aragon et de Groeth ${ }^{32}$ "absolument convaincu[s] que la Finlande a lâchement attaqué l'URSS ", de Gide qui "n'a pas encore dit un mot de la guerre », du

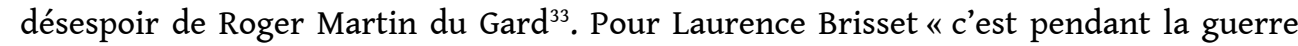
que la position stratégique de Paulhan se révèle dans toute son évidence: son réseau unique de relations le rend incontournable $»^{34}$. Fidèle à la mission qu'il s'est donnée, il fait savoir à Denis de Rougemont ce qu'il sait, prêt à se rendre utile. Et de citer Lie Tseu : «Qui se refuse à faire la guerre, la faisant pourtant, gagne la guerre ». Malgré la relance sur une note attendue et sur le chapitre des Personnes du drame à publier dans la $N R F$, le ton de cette lettre a déjà un avant-goût de la débâcle. Le numéro de juin sera le dernier à paraître et en juillet, les éditeurs quittent Paris.

Nous connaissons l'histoire de la revue pendant l'occupation et l'hésitation de Paulhan de participer à la NRF de Drieu La Rochelle. La revue reparait rapidement, en décembre 1940, et le rôle de Paulhan sera "complexe »: trop attaché à la NRF pour la voir disparaître, ayant choisi «son camp, celui de l'espoir et du silence $»^{35}$ dès avant la reparution de la revue, il sera singulièrement indulgent - ou aveugle - face à Drieu, qu'il préfèrera surveiller de près en acceptant d'abord de corriger toutes les épreuves, puis en entrant dans un comité de rédaction réunissant Arland, Jouhandeau, Giono, Montherlant et Drieu ${ }^{36}$.

\section{La reprise du dialogue : la correspondance d'après- guerre}

L'après-guerre marque le départ d'une seconde correspondance, sporadique et sans doute incomplète, témoignage d'une période difficile pour les deux hommes mais aussi d'une grande connivence sur certains sujets.

Dans une lettre du 17 juillet 1947, Rougemont exprime sa reconnaissance envers Paulhan qui était venu lui rendre visite à Paris. Il lui rapporta des pages de De la paille et $d u$ grain, et Denis de Rougemont lui saura gré du réconfort que cette visite et les textes de Paulhan lui ont apporté :

[...] vos textes sont ce que le mot France représente quand je me le dis ou le pense, parce qu'ils le sont sans le dire, comme invisiblement, donc sans me gêner un seul instant. Toutes les «manifestations françaises » m'éloignent, faussent la France à mes yeux, me font douter de (ou m'interroger sur) mes raisons de l'aimer. Dans vos textes je me sens rassuré ${ }^{37}$. 


\subsection{Débats sur l'Europe}

26 des préoccupations " autrement plus urgentes " que les notes destinées à la Nouvelle Revue française, bien que Paulhan, avant de reprendre finalement la revue en 1953 avec Marcel Arland, parvienne à enrôler Rougemont dans deux numéros des Cahiers de la Pléiade $^{38}$, en 1948, et en 1950 dans un numéro consacré à Saint-John Perse ${ }^{39}$. Ce dernier texte fut envoyé par Rougemont alors en pleins préparatifs de la Conférence européenne de la culture qui se tint à Lausanne du 8 au 12 décembre $1949^{40}$. En ces années d'effervescence européenne, beaucoup d'espoir et de confiance mutuelle se sentent encore dans ces lettres entre les deux écrivains. Paulhan affirme qu'il «serait plus tranquille » si Rougemont était «à Strasbourg, à la place de ces 87 parlementaires $\|^{41}$, alors que Rougemont vient de lui envoyer la circulaire destinée à de nombreux intellectuels, exposant les buts de la Conférence européenne de la culture en préparation. Malgré l'attaque de Paulhan contre les fédéralistes, parue dans Liberté de l'Esprit ${ }^{42}$, Rougemont l'enjoint à être «avec nous $»^{43}$ et tente de répondre à ses arguments dans le numéro d'avril $1950^{44}$. Mais Paulhan ne répond plus présent et dans une lettre dont le style un peu télégraphique s'explique aussi par les troubles oculaires dont il souffre, le désaccord ne fait que s'approfondir: il affirme avoir préféré Rougemont "mondialiste », et regrette qu'il ne le soit resté longtemps ${ }^{45}$. La réponse ne tarde pas et elle arrive dactylographiée, adressée du Palais Wilson "à Monsieur Jean Paulhan " sur le papier à en-tête du Bureau d'études pour un Centre européen de la culture, ouvert par Rougemont un an auparavant. Ce détail ne fait que souligner symboliquement la nouvelle évolution et la «réorientation de l'itinéraire de l'écrivain $»^{46}$. C'est peut-être aussi «faute d'obtenir des relais, des points d'ancrage dans les milieux littéraires parisiens ${ }^{47}$, que Denis de Rougemont se réoriente dans l'action culturelle. Mais si nous pouvons voir là «une sorte de reconversion partiellement conforme à son profil d'homme de lettres ${ }^{48}$, ce qui semble constituer un tournant dans le parcours d'écrivain n'est en réalité que l'accomplissement d'une trajectoire intellectuelle : la posture littéraire définie comme « la manière singulière d'occuper une " " position " dans le champ littéraire ${ }^{49}$ s'est peut-être modifiée, mais le tournant s'est opéré dans le champ littéraire lui-même et la "posture intellectuelle » demeure très conséquente, presque immuable depuis les années trente. S'il ne voit « pas très bien l'utilité d'une duplique » de la part de Paulhan, Rougemont rappelle ici encore une fois que le terme fédéraliste désigne une tendance très définie parmi ceux qui veulent unir les peuples européens et ne peut s'étendre à tous ceux «qui ont un jour, et vaguement, parlé d'union $»^{50}$. Le ton est cinglant et les lettres suivantes de Paulhan attestent de son scepticisme grandissant, même lorsqu'il se veut conciliant. Suite probablement à la réception des Lettres aux députés européens, il se montre encore plus pessimiste dans une missive datée du 10 septembre 1950. "Pas un des hommes de Strasbourg ne veut l'Europe », dit-il. Et il poursuit en post-scriptum :

On ne s'accorde pas, parce qu'on se dit « nous allons bien nous accorder, ce sera très utile, etc. " Non, on a une foi commune, on est prêt à mourir pour une même cause - et il se trouve aussi que l'on s'est accordé (sans l'avoir fait exprès).

Or la souveraineté mondiale peut être une cause, l'Europe - à tort ou à raison - n'en est pas une : ce n'est pour le moment qu'une affaire d'armée et de généraux ${ }^{51}$.

La subtilité et la complexité de la personnalité de Paulhan furent l'objet de plusieurs analyses. Nous ne nous étonnerons point de voir souvent dans ces lettres les 
informations les plus importantes arriver en post-scriptum, et Denis de Rougemont semble s'adapter avec aisance à cet habitus, l'adoptant même parfois. Ainsi ajoutera-t-il en post-scriptum une remarque attestant son profond désir de figurer au sommaire du numéro commémoratif consacré à Gide ${ }^{52}$.

\subsection{Derniers échanges : Gide, Saint-John Perse...} la NRF, d'un chapitre ${ }^{55}$ - «des pages bien fortes $»^{56}$, déclare Paulhan -, de L'aventure occidentale de l'homme, qui doit paraitre chez Albin Michel en 1957, cette seconde «liasse » contient encore quelques échanges sur Saint-John Perse par exemple. Denis de Rougemont fait part des difficultés de "Léger » avant que celui-ci n'obtienne le prix Nobel en 1960, mais Paulhan, qui entretient une correspondance ${ }^{57}$ soutenue avec l'auteur d'Amers, est bien au courant de l'installation de celui-ci dans la presqu'île de Giens.

En 1959, Jean Paulhan félicite Denis de Rougemont pour un article paru dans Preuves ${ }^{58}$, qui n'est pas sans lui rappeler la première note de Rougemont publiée en 1931 dans la $N R F^{59}$. Puis en 1961, la revue donnera encore un long extrait de Comme toi-même $e^{60}$. factuelles et souvent aussi très personnelles sur la vie intellectuelle de la France des années 1930-1960, par cette citation tirée d'une des dernières lettres de Jean Paulhan adressée à Denis de Rougemont, qui pourrait presque en être la devise :

Que devenez-vous, et quand m'enverrez-vous un texte pour la nrf? Bientôt, n'est-ce pas? Et où en est l'Europe? Je n'ai pas le sentiment qu'elle ait fait de grands progrès, depuis notre discussion. Mais là-dessus vous en savez plus que moi.

À vous, avec amitié.

Jean $\mathrm{P}^{61}$. 


\section{BIBLIOGRAPHIE}

ACKERMANN, Bruno, Denis de Rougemont : une biographie intellectuelle, Genève, Labor et Fides, 2 vols, 1996.

BRISSET, Laurence, La NRF de Paulhan, Paris, Gallimard, 2003.

CORBELLARI, Alain, « Denis de Rougemont : un comploteur protestant à la NRF », in Daniel Magetti (dir.), Les écrivains suisses et La Nouvelle Revue Françaises, Paris, Éditions Classiques Garnier, 2009, p. 77-94.

GARDES-TAMINE, Joëlle (éd.), Correspondance Saint-John Perse / Jean Paulhan (1925-1966), Paris, Gallimard, 1991.

GENETTE, Gérard, Seuils, Paris, Éditions du Seuil, 1987.

GUÉRIN, Jeanyves (dir.), La Nouvelle Revue Française de Jean Paulhan, 1925-1940 et 1953-1968, Actes du colloque de Marne-la-Valée, Paris, Éditions Le Manuscrit, 2006.

HÉRIARD-DUBREUIL, Emmanuelle, « Mouvements personnalistes des années 1930 : Denis de Rougemont médiateur », in Olivier Dard, Étienne Deschamps (dir.), Les relèves en Europe d'un aprèsguerre à l'autre, Bruxelles, Peter Lang, 2005, p. 217-231.

MEIzOZ, Jérôme, Postures littéraires : mises en scène modernes de l'auteur, Genève, Slatkine, 2007. PAULHAN, Jean, Flower John Ernest (éd.), Autour de la Lettre aux directeurs de la Résistance de Jean Paulhan, Presses universitaires de Bordeaux, 2003.

RougEmont, Denis de, « Adieu, beau désordre », Revue de Genève, 214 (mars 1926), p. 311-319.

-, «À prendre ou à tuer (Cahier de revendications) ", Nouvelle Revue française, 231 (décembre 1932), p. 838-845.

-, «Sur un certain front unique », Europe, 122 (15 février 1933), p. 303-304.

-, «Les Éléments de la grandeur humaine, par Rudolf Kassner », Nouvelle Revue française, 217 (octobre 1931), p. 640-643.

—, « Note sur la voie clandestine », Cahiers de la Pléiade, hiver 1948, p. 57-63.

—, « Saint-John Perse et l'Amérique », Cahier de la Pléiade, été-automne 1950, p. 136-139.

—, « La découverte du temps ou l'aventure occidentale », Nouvelle Revue française, 51 (mars 1957), p. $400-415$.

-, « Rudolf Kassner et la grandeur », Preuves, 100 (juin 1959), p. 63-71.

-, « La personne, l'ange et l'absolu ou le dialogue Occident-Orient », Nouvelle Revue française, 100 (avril 1961), p. 585-617.

STENGER, Nicolas, Denis de Rougemont : les intellectuels et l'Europe au XXe siècle, Presses universitaires de Rennes, 2015.

TREBITSCH, Michel, « Le front commun de la jeunesse intellectuelle. Le Cahier de revendications de décembre 1932 », in Gilbert Merlio (dir.), Ni gauche, ni droite : les chassés-croisés idéologiques des intellectuels français et allemands dans l'entre-deux-guerres, Bordeaux, Éditions de la Maison des sciences de l'homme d'Aquitaine, 1995, p. 209-227. 


\section{NOTES}

1. G. Genette, Seuils, p. 343.

2. Ainsi par exemple J. Guérin (dir.), La Nouvelle Revue Française de Jean Paulhan, 1925-1940 et 1953-1968.

3. Lettre de Jean Paulhan à Denis de Rougemont, le 3 mai 1926 (BPUN, Fonds DdR, ID2256).

4. D. de Rougemont, « Adieu, beau désordre».

5. L. Brisset, La NRF de Paulhan, p. 39.

6. Ibid., p. 219.

7. Seul un brouillon a été conservé : Lettre de Denis de Rougemont à Jean Paulhan, le 2 juin 1926 (BPUN, Fonds DdR, ID1332).

8. A. Corbellari, « Denis de Rougemont : un comploteur protestant à la NRF », p. 77.

9. M. Trebitsch, « Le front commun de la jeunesse intellectuelle», p. 213

10. Lettre de Jean Paulhan à Denis de Rougemont, le 30 septembre 1932 (BPUN, Fonds DdR, ID2256).

11. Sur le contexte de la rédaction de ce numéro, voir E. Hériard-Dubreuil, « Mouvements personnalistes des années $1930 »$.

12. Le sommaire comprendra les contributions de Henri Lefebvre, Paul Nizan, Philippe Lamour, Jean Sylveire, Thierry Maulnier, Arnaud Dandieu et Claude Chevalley, Emmanuel Mounier, Georges Izard, René Dupuis et Alexandre Marc, Robert Aron.

13. Lettre de Jean Paulhan à Denis de Rougemont, le 9 décembre 1932 (BPUN, Fonds DdR, ID2256).

14. Lettre de Denis de Rougemont à Jean Paulhan, décembre 1932 (IMEC, Fonds Paulhan).

15. D. de Rougemont, « Sur un certain front unique », p. 303 sq.

16. Cette "passe d'arme» est présentée en détail par Michel Trebitsch qui voit dans les accusations de Nizan «l'archétype de toute l'argumentation ultérieure contre l'idéologie de la révolution spirituelle » (cf. « Le front commun de la jeunesse intellectuelle », p. 218 sq.).

17. Lettre de Denis de Rougemont à Jean Paulhan, le $1^{\text {er }}$ février 1933 (IMEC, Fonds Paulhan).

18. La violence de cette postface, spirituelle et verbale, est clairement assumée : la rupture se veut définitive avec le marxisme se révélant «comme un cas privilégié de la folie capitalistematérialiste ", et Rougemont prévoit même la préparation " en sous-main de terrains d'entente » entre les capitalistes et l'URSS. D. de Rougemont, "À prendre ou à tuer (Cahier de revendications) ».

19. Lettre de Jean Paulhan à Denis de Rougemont, le 7 juillet 1933 (BPUN, Fonds DdR, ID2256).

20. Lettre de Denis de Rougemont à Jean Paulhan, le 4 octobre 1933 (IMEC, Fonds Paulhan).

21. B. Ackermann, Denis de Rougemont, p. 410.

22. Lettre Jean Paulhan à Denis de Rougemont, novembre 1933 (BPUN, Fonds DdR, ID2256).

23. Lettre de Denis de Rougemont à Jean Paulhan, le 15 mars 1934 (IMEC, Fonds Paulhan). Sur l'histoire et la stratégie de L'Ordre nouveau, voir M. Trebitsch, « Le front commun de la jeunesse intellectuelle », p. 216-218 et B. Ackermann, Denis de Rougemont, p. 250-309. Sur l'engagement personnaliste de Denis de Rougemont, voir N. Stenger, Denis de Rougemont, p. 38 ss.

24. Lettre de Denis de Rougemont à Jean Paulhan, le 11 novembre 1933 (IMEC, Fonds Paulhan).

25. La Vision physiognomonique du monde sera refusée par Gallimard, ce que Paulhan, sensible à la situation de Rougemont, ne lui communiquera que quatre mois plus tard : Lettre de Jean Paulhan à Denis de Rougemont, le 17 août 1934 (BPUN, Fonds DdR, ID2256).

26. Lettre de Denis de Rougemont à Jean Paulhan, le 6 novembre 1935 (IMEC, Fonds Paulhan).

27. B. Ackermann, Denis de Rougemont, p. 574.

28. Lettre de Jean Paulhan à Denis de Rougemont, sans date (probablement 1938) (BPUN, Fonds DdR, ID2256).

29. L. Brisset, La NRF de Paulhan, p. 317. 
30. Terme utilisé par Rougemont dans une lettre à Brice Parain, le $1^{\text {er }}$ septembre 1938. B. Ackermann, Denis de Rougemont, p. 574.

31. Grandeur ou Importance de Gide furent des titres abandonnés, et son très remarqué «Au sujet du Journal d'André Gide » paraîtra finalement dans la NRF du $1^{\text {er }}$ décembre 1940.

32. Bernard Groethuysen.

33. Lettre de Jean Paulhan à Denis de Rougemont, le 22 janvier 1940 (BPUN, Fonds DdR, ID2256).

34. L. Brisset, La NRF de Paulhan, p. 199.

35. Ibid., p. 93.

36. Ibid., p. 97.

37. Lettre de Denis de Rougemont à Jean Paulhan, le 7 juillet 1947 (IMEC, Fonds Paulhan).

38. D. de Rougemont, « Note sur la voie clandestine».

39. D. de Rougemont, « Saint-John Perse et l'Amérique».

40. Sur l'engagement européen de Denis de Rougemont et le contexte de la naissance du Centre européen de la culture, voir B. Ackermann, Denis de Rougemont, p. 860-863, et N. Stenger, Denis de Rougemont, p. 133-150.

41. Il s'agit des députés du Conseil de l'Europe, créé le 5 mai 1949.

42. J. Paulhan, « Lettre aux directeurs sur l'Europe », p. 155-157.

43. Lettre de Denis de Rougemont à Jean Paulhan, le 26 janvier 1950 (IMEC, Fonds Paulhan).

44. D. de Rougemont, « Un gage à Jean Paulhan!», p. 33 sq.

45. Lettre de Jean Paulhan à Denis de Rougemont, début 1950 (BPUN, Fonds DdR, ID2256).

46. Nicolas Stenger, Denis de Rougemont, p. 167.

47. Ibid.

48. Ibid.

49. J. Meizoz, Postures littéraires, p. 18.

50. Lettre de Denis de Rougemont à Jean Paulhan, le 24 mars 1950 (IMEC, Fonds Paulhan).

51. Lettre de Jean Paulhan à Denis de Rougemont, le 10 septembre 1950 (BPUN, Fonds DdR, ID2256).

52. Lettre de Denis de Rougemont à Jean Paulhan, le 18 septembre 1951 (IMEC, Fonds Paulhan).

53. Ibid.

54. A. Corbellari, « Denis de Rougemont », p. 82.

55. D. de Rougemont, « La découverte du temps ou l'aventure occidentale ».

56. Lettre de Jean Paulhan à Denis de Rougemont, 18 décembre 1956 (BPUN, Fonds DdR, ID2256).

57. J. Gardes-Tamine (éd.), Correspondance Saint-John Perse / Jean Paulhan (1925-1966).

58. D. Rougemont, « Rudolf Kassner et la grandeur ».

59. D. Rougemont, « Les Éléments de la grandeur humaine, par Rudolf Kassner ».

60. D. de Rougemont, « La personne, l'ange et l'absolu ou le dialogue Occident-Orient ».

61. Lettre de Jean Paulhan à Denis de Rougemont, 1959 (BPUN, Fonds DdR, ID2256).

\section{RÉSUMÉS}

La correspondance d'écrivains, à la croisée de l'intime et du social, constitue un corpus précieux pour le critique à la recherche d'informations venant éclairer l'œuvre et sa genèse, l'auteur, l'époque. Outre l'intérêt proprement biographique, d'un point de vue endogénétique, elle met en lumière les différentes phases d'élaboration d'un texte, du projet à la réalisation, le choix d'un 
style et les réactions critiques. L'importance de cette fonction paratextuelle ${ }^{1}$ est accrue dans le cas de la correspondance d'un directeur de revue et de l'un de ses collaborateurs, à l'intérêt biographique venant s'ajouter l'étude de champs. La correspondance de Denis de Rougemont et de Jean Paulhan, si courte soit-elle, constitue ainsi une mine d'informations sur la vie intellectuelle, l'édition et la presse critique de la France des années 1930-1960.

\section{AUTEUR}

\section{KRISZTINA HORVÁTH}

Université Eötvös Loránd de Budapest 